\section{World-Wide Web Survey Research Made Easy with WWW Survey Assistant}

WWW Survey Assistant is a utility that simplifies World-Wide Web (WWW, or Web) survey research by custom-creating an HTML (hypertext markup language) form document to present computerized questionnaires and tests, and custom-generating a sophisticated CGI (common gateway interface) program for accepting data from the form. No CGI programming or HTML experience on the part of the user is necessary, thereby making advanced WWW survey research accessible to researchers without such skills.

An important component of the ease of survey creation with WWW Survey Assistant is its on-line Web editors, which allow survey administrators to build and test their questionnaire over the Web by using their favorite graphical Web browser. This software announcement details features of WWW Survey Assistant that are specially designed for sophisticated survey research on the Web, and briefly explains the steps that one takes on line to create an interactive Web survey using this utility.

Features of the CGI program. Although there are many advantages to using the Web for survey research (see Schmidt, 1997), perhaps the most unique characteristic of this medium is the ability to implement interactive or dynamic surveys. Interactive surveys present the user with specialized feedback that is computed on line. Such information can be based on the respondent's particular answers, or it can consist of summary data based on an entire group of respondent data. Because a CGI program is required to manipulate survey data on line, and to prepare and present feedback to the respondent, the CGI program must contain the feedback information and formulae pertaining to the derivation of such information. To accomplish this feat, programming skills are ordinarily necessary on the part of the survey administrator. However, with WWW Survey Assistant, the survey administrator supplies formulae, information about the reply, and/or actions to take, and Survey Assistant writes the appropriate CGI program commands. For test questions, as opposed to survey questions, the administrator supplies specialized feedback to be presented for correct and incorrect responses.

A second feature of the Survey Assistant CGI program and the document containing the survey is that the HTML of both are fully customizable. A variety of different question presentation formats are available, so there is freedom to design questionnaires to suit specific research goals. Presentation formats for questions with mutually exclusive responses are available, as are formats for questions that allow multiple responses. Stand-alone graphics or sound, as well as graphic and text links, can be incorporated anywhere, as can different font sizes, colors, and styles.
As one might guess from the preceding information, the CGI program is the most important component of WWW survey research (see Kieley, 1996; Schmidt, 1997). Because WWW Survey Assistant's CGI program is expressly created for scientific use, it has a number of amenities to ensure that data integrity is maintained. For instance, administrators may select whether responses to all questions, or only to specific questions, are required. Should a user fail to supply a response to a required question, a customizable message can be presented along with a list of unanswered items.

WWW Survey Assistant provides survey administrators with the ability to automatically check the data that they receive. The administrator supplies acceptable types and values for a given response (i.e., numeric vs. textual input, or a range of legal values), and the CGI program has the ability to ensure that the user has entered appropriate information. As is the case for missing data, if a user fails to supply an adequate response, a customizable message is presented along with an explanation of the range of values that is expected.

The CGI programs generated are security aware: They can ensure that the server origin of the questionnaire form is authorized. Data submitted from forms that are dispensed from unauthorized Web servers are suspect (see Schmidt, 1997). The default option is to have the generated survey installed on the Survey Assistant server; this frees users from the need to investigate operating their own Web server and requiring them to install the CGI program and the utilities that it needs. Users can then visit the Survey Assistant server and access their survey and data files by way of a password-protected utility. For the more technically inclined, or for users who want more control over their setup, the CGI program (which is written in the freely available Perl5 language, and which works under UNIX, Macintosh MacOS, and Microsoft Windows) can be acquired for installation on the survey administrator's own Web server.

The on-line WWW Survey Assistant editor. There are two methods for editing surveys on line: one is directed at advanced users and the other at the novice. The remainder of this announcement deals conceptually with the steps required to create a simple Web survey using the on-line novice Web editor.

Users first log into the editor accessed through the Web site located at http://or.psychology.dal.ca/ wcs by supplying any (one word) user name, project name, and password. The user name identifies all of a given user's work, and the project name identifies the particular project to be edited during a given session. Users may select an example survey to edit, or enter the editor with a blank project.

After logging in, the user's browser window is split into two frames (with frames-capable browsers-currently, Netscape's Mozilla 2.0 or greater, and Microsoft's Inter- 
net Explorer 3.0 or greater support this feature). The left frame is the "Action Selector" window; from this, users can do things such as add survey or test questions, graphics, text, links, etc. The right-hand window is the response window. Actions that the user takes to edit the survey will take place in the response window. The Action Selector remains present throughout the Web editing session (browsers that do not support frames use the same window to alternately display the Action Selector and the response.)

All Survey Assistant projects consist of three main components: the SURVEY page, the REPLY page, and the INCOMPLETE page. The SURVEY Web page contains the survey or test questions. To move on, the respondent fills out the SURVEY page and clicks on the submit button. If all of the questions have been filled in adequately, the REPLY page is presented along with feedback (prespecified responses based on correct or incorrect answers to test questions, the results of formulae computed on collected information, a breakdown of the data acquired to date for a given variable, etc.); otherwise, the INCOMPLETE page is displayed. The INCOMPLETE page is the perfect place to list questions that were missed or inappropriately filled out.

The Survey Editor allows users to assemble different modules or "chunks" in order to create a survey. Each chunk corresponds to an action (such as adding survey or test questions, images, links, etc). Initially, the list of chunks includes only the SURVEY (chunk 0), REPLY (chunk 1), and INCOMPLETE (chunk 2) chunks. To create a survey or test, users are required to insert information to be presented (i.e., plain text, questions, links, feedback, etc.) after each of these sections.

At the top of the Action Selector is a pop-up menu with all of the different editing actions that the user can take. Beneath are two string inputs with the text "Target Chunk" and "Label" in front of them. Use the Action Selector to specify what action is to be taken, and on what chunk this action is to apply. The label field allows the user to associate an arbitrary string with the chunk. Click on the Execute Action button, and a request for further information specific to the action selected will appear in the response window. If the action involves adding a new chunk, that chunk will be numbered according to the value supplied in the Action Selector window. The editor ensures that users cannot do anything illegal, such as add questions to the REPLY section (recall that only the SURVEY component allows questions).

The lower portion of the Action Selector contains a number of clickable buttons. Users can click on "Update Survey" to examine and use the survey being edited (both the HTML and the CGI program output are produced to be inspected and tested). Following the "Your Survey" link allows users to view the survey and test the CGI on line! Clicking on the "Show Listing" button presents the current listing of the chunk components that have been assembled, along with their associated chunk numbers. Clicking on the "Instructions" button presents a rudimentary overview of the editing process and describes the actions available in the Web editor. The "Backup" button allows users to make an archive of their project that can be downloaded to their local system for storage. The "Restore" feature of the editor allows users to upload an archive and continue to work on it at a later time. These features are important, because the survey work of unlicensed users is automatically erased from the survey server after about 2 weeks.

The "Install Survey" button publishes the survey by creating a special version and installing it on the Survey Assistant Web server. The URL and a link to the installed version of the survey is presented. If users wish to install the CGI program on their own Web server, they can click on the "Acquire Survey CGI" button and after answering some server-specific questions and upon payment of an acquisition fee, they will be e-mailed the finished version of their HTML document and CGI program, along with detailed installation instructions.

Summary. The goal of this software announcement has been to familiarize readers with the ease with which one can create a Web test or survey by using WWW Survey Assistant. There is a great amount of flexibility in the types of documents that one can present and in the functionality that can be incorporated into the CGI program without having to learn how to program CGIs. Even more capabilities of the generated CGI can be revealed by examining the options and switches that have not been adopted or discussed in this paper (i.e., features such as security options, summarizing received data, handling multiple-response questions, etc.), or by reading the documentation and tutorials available at the WWW Survey Assistant Web site.

WWW Survey Assistant and information pertinent to publishing surveys on the World-Wide Web can be found at http://or.psychology.dal.ca/ wcs.

\section{REFERENCES}

KIELEY, J. M. (1996). CGI scripts: Gateways to World-Wide Web power. Behavior Research Methods, Instruments, \& Computers, 28, 165-169. SchмidT, W. C. (1996). World-Wide Web survey research: Benefits, potential problems, and solutions. Behavior Research Methods, Instruments, \& Computers, 29, 274-279.

William C. Schmidt Dalhousie University

(Manuscript received September 23, 1996; revision accepted for publication December 18, 1996.) 